\title{
Intracranial ependymoma in children: current status and future trends on diagnosis and management
}

\author{
Frederick A. Boop • Spyros Sgouros
}

Received: 23 June 2009 / Published online: 21 July 2009

(C) Springer-Verlag 2009

\begin{abstract}
"In the game of pediatric brain tumors, there is only one way to win. That is to remove all of the tumor and to leave the child without a deficit. If the neurosurgeon accomplishes anything less than this, he has not won the game"
\end{abstract}

C. Raffel

One of the most exciting elements of the practice of pediatric neurosurgery has been the advances made in the realm of neuro-oncology over the past three decades. Moving from a time in which brain tumors were diagnosed primarily on clinical grounds, the understanding of cellular and molecular events were based on the appearance of paraffin-embedded specimens viewed under glass, the

\footnotetext{
F. A. Boop $(\bowtie)$

Department of Neurosurgery,

University of Tennessee Health Science Center,

Memphis, TN, USA

e-mail: faboop@aol.com

F. A. Boop

LeBonheur Children's Hospital,

Memphis, TN, USA

F. A. Boop

St. Jude Children's Research Hospital,

Memphis, TN, USA

F. A. Boop

Semmes-Murphey Clinic,

Memphis, TN, USA

\section{S. Sgouros $(\bowtie)$}

Department of Neurosurgery, "Attikon” University Hospital, University of Athens,

Athens, Greece
}

radiological tools necessary to define the extent of the disease were rudimentary, and the surgical technology available to make tumor removal safe and to offer the survivor a reasonable quality of life were rudimentary, we have now moved into a fast-paced arena in which each year brings new research with better understanding of tumor genomics, tumor suppressors and promotors, stem cells, vascular supply, etc. Advances in neuroimaging now allow accurate preoperative diagnosis and staging, image-guided surgery offers improved operative planning and execution, and better postoperative surveillance tools allow earlier detection and more effective treatments for recurrent disease. More sensitive tools now allow earlier detection of secondary events such as learning disabilities, quality of life measures, and hormonal deficits, which can be treated effectively through improved pharmacology. No area of neuro-oncology serves as a better model for these statements than does the treatment of childhood ependymoma. It is anticipated that this compendium will offer current knowledge on the status of ependymoma therapy for the neurosurgeon, that it will correct certain misperceptions related to ependymoma therapy, and that it will give direction for future advances in the treatment of this disease. I would like to thank in advance all of the contributors who spent long hours working on their manuscripts for this Supplement. That those asked to contribute are world leaders in the field of pediatric neuro-oncology becomes apparent as one peruses the table of contents of this journal.

There are a number of truths which we can now apply to pediatric ependymoma. First is that in $90 \%$ of cases, the disease will be localized at presentation. At that stage, the focal disease process will usually respond to focal treatments [1]. Thus, the days of craniospinal irradiation or even whole brain irradiation for ependymomas should be well 
behind us. Second, numerous prospective controlled trials from around the world have shown that ependymoma is still a surgical disease. What we accomplish as neurosurgeons in the resection of these tumors is the most significant determinant of outcome [2]. With ependymoma, if the neurosurgeon retreats from surgery leaving tumor behind and telling himself that he must "first do no harm", he has ultimately created the greatest harm to that patient. Thus, if a neurosurgeon feels a priori that he is not likely to be able to completely resect a focal ependymoma, he owes it to his patient to consider referral of that patient to a specialty center in which better tools and more experience might afford the child a better chance at survival. To partially resect an ependymoma and then refer the child for adjuvant therapy is doomed to fail. Third, early postoperative high-quality magnetic resonance imaging (MRI) has proven invaluable in managing these patients. Controlled studies have shown that even when the surgeon believes he has accomplished a gross total resection, that postoperative imaging may prove him wrong in as much as a third of the cases [3]. Postoperative computed tomography (CT) scanning is not sufficient to detect small pieces of resectable residual tumor. Parents should be made aware before the initial surgery that their child will receive a postoperative MRI study and that the finding of significant residual tumor will prompt a return to the operating room for an attempt at removal of as much residual tumor as is safely possible.

It has been shown also that ependymomas are generally sensitive to radiation therapy. Since the majority of ependymomas are focal at presentation, following surgical resection with focal irradiation will significantly reduce the chances of recurrence, either locally or with disseminated disease. That a number of children with (1) supratentorial ependymoma or spinal ependymoma and (2) differentiated histology can be cured by gross total resection alone has been suggested but current case series are too small to know how to best select these cases and what risk factors will predict recurrence [4]. This is an area which clearly requires more attention.

The work of Taylor et al., reviewed in this issue, have shown that supratentorial, posterior fossa, spinal, and myxopapillary ependymoma each represent genetically different tumors [5]. In the past, it had also been stated that histology was not a significant determinant of outcome in pediatric ependymoma. This was certainly true in case series in which a gross total resection was performed in a minority of patients. Children with both differentiated (WHO II) and anaplastic (WHO III) tumors did equally poorly when neither were completely resected. But over time, as our surgical tools have allowed a gross total resection to be accomplished in the vast majority of children, confirmed by postoperative MRI, we do see a significant difference in outcome of children favoring those with lower-grade histology [6]. Finally, advances in radiation therapy now allow more targeted therapy at well-established doses for ependymoma. Under-dosing contributes to higher failure rates. Likewise, proton beam therapy and three-dimensional conformal treatment have led to more effective treatment with sparing of adjacent functional structures, thus allowing us to give focal adjuvant therapy at a younger age and allowing for better quality of life for survivors.

The current role of adjuvant chemotherapy in childhood ependymoma has previously been limited. That treatment with platinum-based chemotherapy agents predisposes these children to greater hearing loss after radiotherapy is well established [6]. Retrospective case series have further shown that for young children with large and vascular tumors, in whom surgical resection is compromised by the risk of exsanguination, two to three rounds of conventional chemotherapy can dramatically reduce the vascularity of ependymomas, making a return to surgery for further resection more safe. The trade-off is that, although less vascular, the tumor may become more fibrous and tenacious after this chemotherapy.

Using standard chemotherapy as treatment for residual disease has not proven fruitful [7]. Although ependymomas may respond transiently, with significant response resulting in delay of early recurrence, the addition of chemotherapy has not proven to extend overall survival for this disease. A phase II randomized Children's Oncology Group trial should open soon to answer this question. Newer biologically active agents are currently under trial and have theoretical advantages, but at present should be limited to carefully constructed clinical trials.

The surgical management of recurrent ependymomas is an area of some controversy, where perhaps the Hippocratic principle of "first do no harm" is increasingly pertinent. As aggressive treatment prolongs survival, more and more children require surgical treatment for recurrent ependymoma, even years after the first surgery [8]. Second and third surgery, especially in the posterior fossa, are associated with increased risk of neurological deficit, as dissection of the lower cranial nerves is more demanding due to arachnoid scarring. The risk of serious side effects, such as bulbar palsy, leading to the need for tracheostomy and gastrostomy are higher after every surgical attempt. Still, the need for a comprehensive attempt of total excision is just as important in recurrent disease as in the first presentation. Once again, the surgical expertise and a multidisciplinary approach to managing such difficult patients are crucial. One could argue that there is even a higher necessity for these children to be treated in specialized centers, where there is a better appreciation of how far one should try to push surgical treatment and when to stop, avoiding a "triumph of technique over reason". 
Finally, it should be recognized that our major improvements in the treatment of childhood ependymoma have only come through the willingness of pediatric neurosurgeons and neuro-oncologists to retrieve fresh tumor tissue at each operation and to cooperate in the enrollment of these patients in prospective clinical trials. Childhood ependymomas occur so infrequently and our discipline changes so rapidly; hence, it is only through participation in clinical trials that we as pediatric neurosurgeons can continue to reduce the burden of this disease.

\section{References}

1. Merchant TE, Li C, Xiong X, Kun LE, Boop FA, Sanford RA (2009) Conformal radiation therapy for pediatric ependymoma. Lancet Oncol 10(3):258-266

2. Pollack IF, Gerszten PC, Martinez AJ, Lo KH, Shultz B, Albright AL, Janosky J, Deutsch M (1995) Intracranial ependymomas of childhood: long-term outcome and prognostic factors. Neurosurgery 37:655-666
3. Dirven CMF, Mooij JJA, Molenaar WM (1997) Cerebellar pilocytic astrocytoma: a treatment protocol based upon analysis of 73 cases and a review of the literature. Child's Nerv Syst 13:17-23

4. Hutkin J, Epstein F, Lefton D et al (1998) Treatment of intracranial ependymoma by surgery alone. Pediatr Neurosurg 29:40-45

5. Taylor MD, Poppleton H, Fuller C, Su X, Liu Y, Jensen P, Magdelano S, Dalton J, Board J, Macdonald T, Rutka J, Guha A, Gajjar A, Curran T, Giltertson RJ (2005) Radial glia cells are candidate stem cells of ependymoma. Cancer Cell 8:323-335

6. Merchant TE, Mulhern RK, Krasin MJ, Kun LE, Williams T, Li C, Xiong X, Khan RB, Lustig RH, Boop FA, Sanford RA (2004) Preliminary results from a phase II trial of conformal radiation therapy and evaluation of radiation-related CNS effects for pediatric patients with localized ependymoma. J Clin Oncol 22 (15):3156-3162

7. Robertson PL, Zeltzer PM, Boyett JM, Rorke LB, Allen JC, Geyer JR, Stanley P, Li H, Albright AL, McGuire-Cullen P, Finlay JL, Stevens KR Jr, Milstein JM, Packer RJ, Wisoff J (1998) Survival and prognostic factors following radiation therapy and chemotherapy for ependymomas in children: a report of the Children's Cancer Group. J Neurosurg 88:695-703

8. Merchant TE, Boop FA, Kun LE, Sanford RA (2008) A retrospective study of surgery and re-irradiation for recurrent ependymoma. Int J Radiation Oncology Biol Phys 71(1):87-97 